\title{
Demyelinating and ischemic brain diseases: detection algorithm through regular magnetic resonance images
}

D. Castillo

René Samaniego

Y. Jiménez

L. Cuenca

O. Vivanco

M. J. Rodríguez-Álvarez 


\title{
Demyelinating and ischemic brain diseases: detection algorithm through regular magnetic resonance images
}

\author{
D. Castillo *a, René Samaniego ${ }^{\mathrm{c}}$, Y. Jiménez $^{\mathrm{a}}$, L. Cuenca ${ }^{\mathrm{a}}$, O. Vivanco ${ }^{\mathrm{b}}$, M.J. Rodríguez-Álvarez ${ }^{\mathrm{d}}$ \\ ${ }^{a}$ Chemistry and Exact Sciences Department; ${ }^{b}$ Biological Sciences Department ; Universidad Técnica \\ Particular de Loja, Ap. 11-01-608, Loja- Ecuador; ${ }^{c}$ Dept. of Radiology, Hospital UTPL, Loja- \\ Ecuador; ; ${ }^{d}$ Dept. of I3M, Universidad Politécnica de Valencia, Spain
}

\begin{abstract}
This work presents the advance to development of an algorithm for automatic detection of demyelinating lesions and cerebral ischemia through magnetic resonance images, which have contributed in paramount importance in the diagnosis of brain diseases. The sequences of images to be used are T1, T2, and FLAIR.

Brain demyelination lesions occur due to damage of the myelin layer of nerve fibers; and therefore this deterioration is the cause of serious pathologies such as multiple sclerosis (MS), leukodystrophy, disseminated acute encephalomyelitis. Cerebral or cerebrovascular ischemia is the interruption of the blood supply to the brain, thus interrupting; the flow of oxygen and nutrients needed to maintain the functioning of brain cells. The algorithm allows the differentiation between these lesions.
\end{abstract}

Keywords: brain disease, image processing, MRI, demyelinating, ischemia.

\section{INTRODUCTION}

Vascular disorders of the human brain are a common precondition and a relevant factor of influence for some of the most devastating neurological conditions like stroke and dementia globally burdening patients, families, and the health care systems $^{1}$.

For instance, Brain demyelination (BD) lesions occur due to damage of the myelin layer of nerve fibers; and therefore this deterioration is the cause of serious pathologies such as multiple sclerosis (MS) disease in the central nervous system $^{2}$ (CNS), leukodystrophy, and disseminated acute encephalomyelitis.

On the other hand, cerebrovascular ischemia (BI) is the interruption of the blood supply to the brain, thus interrupting; the flow of oxygen and nutrients needed to maintain the functioning of brain cells ${ }^{3}$. It occurs more frequently in older people with an increasing prevalence of important risk factors such diabetes and arterial hypertension, generating focalized manifestations like paralysis, aphasia among others. In summary, ischemia, is produced by a series of physiological and biochemical changes that lead to ischemic necrosis of a given area, dependent on the occluded vessel, in whole or in part ${ }^{4}$.

In this way, all diseases affecting the cerebral vasculature are one of the most relevant global health problems. Currently, the diagnosis of $\mathrm{BD}$ and $\mathrm{BI}$ is based primarily on clinical and neuropsychological assessments ${ }^{5-7}$.

Neuroimaging has also been recognized as a powerful tool to analyze brain changes. However, the analysis of brain images is a difficult task because the spatial pattern of brain degeneration. Consequently, several attempts are being made to develop automated tools that will allow a more sensitive and consistent analysis.

Certainly most of these attempts have focused on the diagnosis of BD from Magnetic resonance imaging. MRI is an imaging technique that produces high quality images of the anatomical structures of the human body, especially in the brain, and provides rich information for clinical diagnosis and biomedical research ${ }^{8,9}$. MRI is the most commonly used imaging modality as it offers high-resolution images in a noninvasive and safe method, without exposing patients to ionizing radiation ${ }^{10}$.

Applications of Digital Image Processing XL, edited by Andrew G. Tescher, Proc. of SPIE Vol. 10396, 103961C · @ 2017 SPIE · CCC code: 0277-786X/17/\$18 · doi: 10.1117/12.2274579 
*dpcastillo@utpl.edu.ec; phone +593 07370 1444; ext.3204; www.utpl.edu.ec

The diagnostic values of MRI are greatly magnified by the automated and accurate classification of the MRI images ${ }^{11,12}$.

As well, the diagnosis of $\mathrm{BD}$ and $\mathrm{BI}$ from MRI is a challenging problem, which received less attention. In the case of T1, T2, and FLAIR images, further investigation is still needed to determine their ability to diagnose BD and BI, especially at the early stage. These images are analyzed to find the number and spatial patterns of the lesions.

Due to the several reports from the Universidad Técnica Particular de Loja Hospital (H-UTPL), in Ecuador there are evidence that several patients suffer brain disorders and many of them suffer pathologies identified as demyelinating (BD) and stroke or ischemia (BI).

The findings of white matter signal changes on magnetic resonance imaging (MRI) brain scan is being increasingly recognized as potentially of clinical significance ${ }^{13}$. Thus, there is an increasing and still unmet need for an even better understanding of $\mathrm{BD}$ and $\mathrm{BI}$ in order to derive better assessment of disease progression and treatment therapeutic efficacy.

For that reason, the principal objective for this project focus on the development of an algorithm for automatic detection of demyelinating and ischemia cerebral lesions through MRIs.

The workflow employed was two stages. First: Design algorithm and test with a semi-automatic process for identify the most relevant features that allow to distingue the BI and BD lesions. Second: The implementation definitively of the algorithm in a software that allow to obtain an automatic process of classification, for that we will use Discrete Wavelet Transform (DWT), principal component analysis (PCA) and a kernel support vector machine (SVM) learning theory will be used to classify all images.

The algorithm developed will allow us the better classification between these two pathologies indicated before BD and BI lesions, the script with the effective validation could be applied for the fast diagnosis and contribute to an effective treatment.

\section{METHODOLOGY}

\section{Dataset}

The proposed approach involved MRI dataset series from $80 \mathrm{H}-\mathrm{UTPL}$ patients that suffers the BI or BD lesions and 100 images of normal patients or suffer another pathology. The images were acquired by RM Philips Achieva 1.5 Teslas. T1, T2-weighted fast spin-echo, and Fast-FLAIR images format were chosen for the analysis.

\section{Design algorithm}

The noise reduction and the elimination of extra cranial brain tissues introduced during MRI acquisition was removed using a python script. It was developed for eliminating the extra cranial brain tissues from T1, T2 and FLAIR images.

Followed by the transformation from DICOM format to a PNG, JPEG, and BMP format. With those formats it is possible to convert the images to a gray scale and select a threshold for to obtain a binary image and extract the features for identify the brain lesions.

In the final algorithm the PNG, JPEG, and BMP format will be used as input in the training algorithm. In the following subsections, we will explain the detailed procedures of the algorithm implementation and the outputs obtained from it. The general overview of the method proposed is shown in Fig.1. 


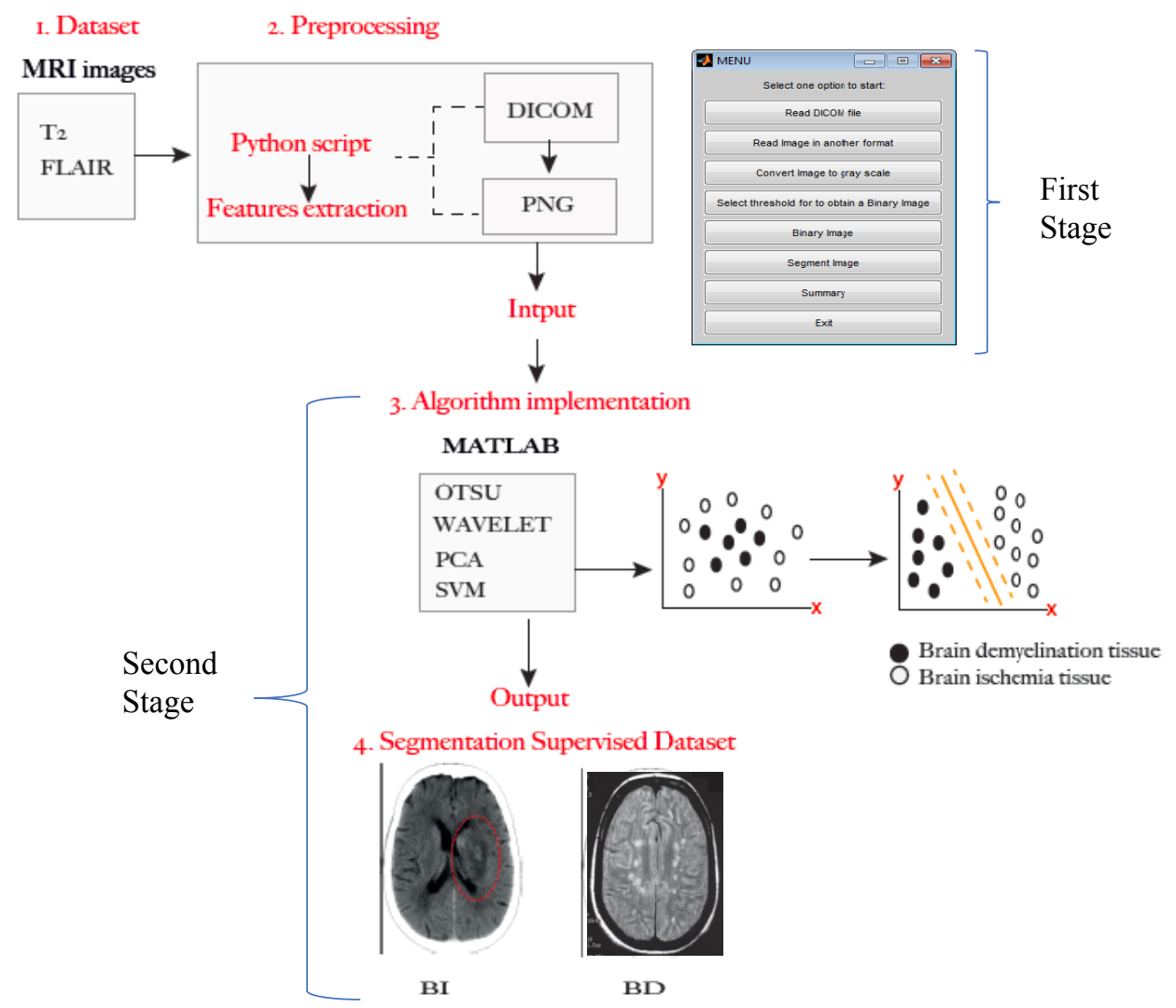

Figure 1. Methodology propose for classification into MRI Images of Brain Ischemia (BI) and Brain demyelination (BD) diseases.

\section{Algorithm implementation}

First instance, the algorithm will be implemented using Matlab R2012a. Applying, OTSU ${ }^{14}$ method to automatically select thresholding for the reduction of a gray level image to a binary image. See table 1.

Table 1. Information and design of algorithm for identify brain ischemia and brain demyelinating diseases into MRI images.

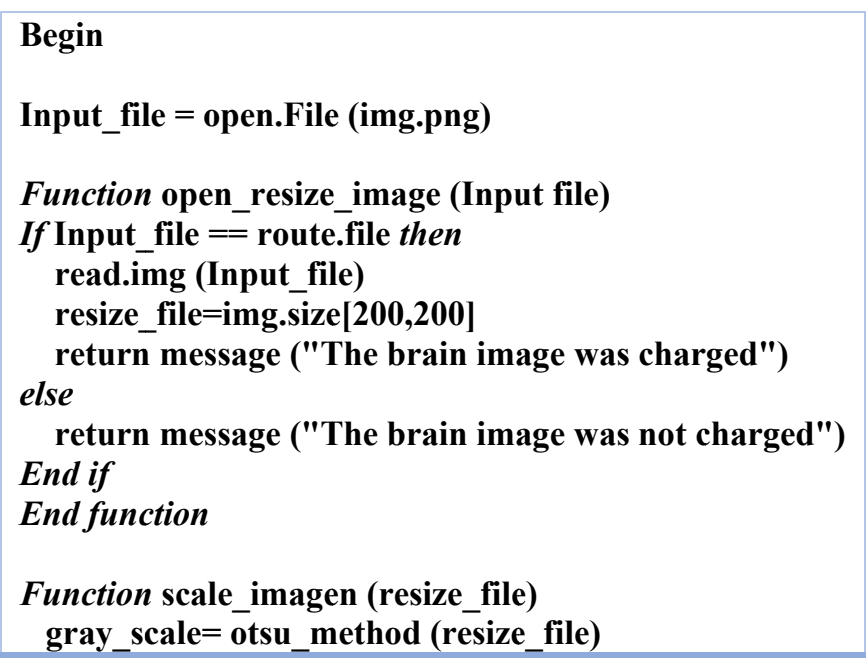




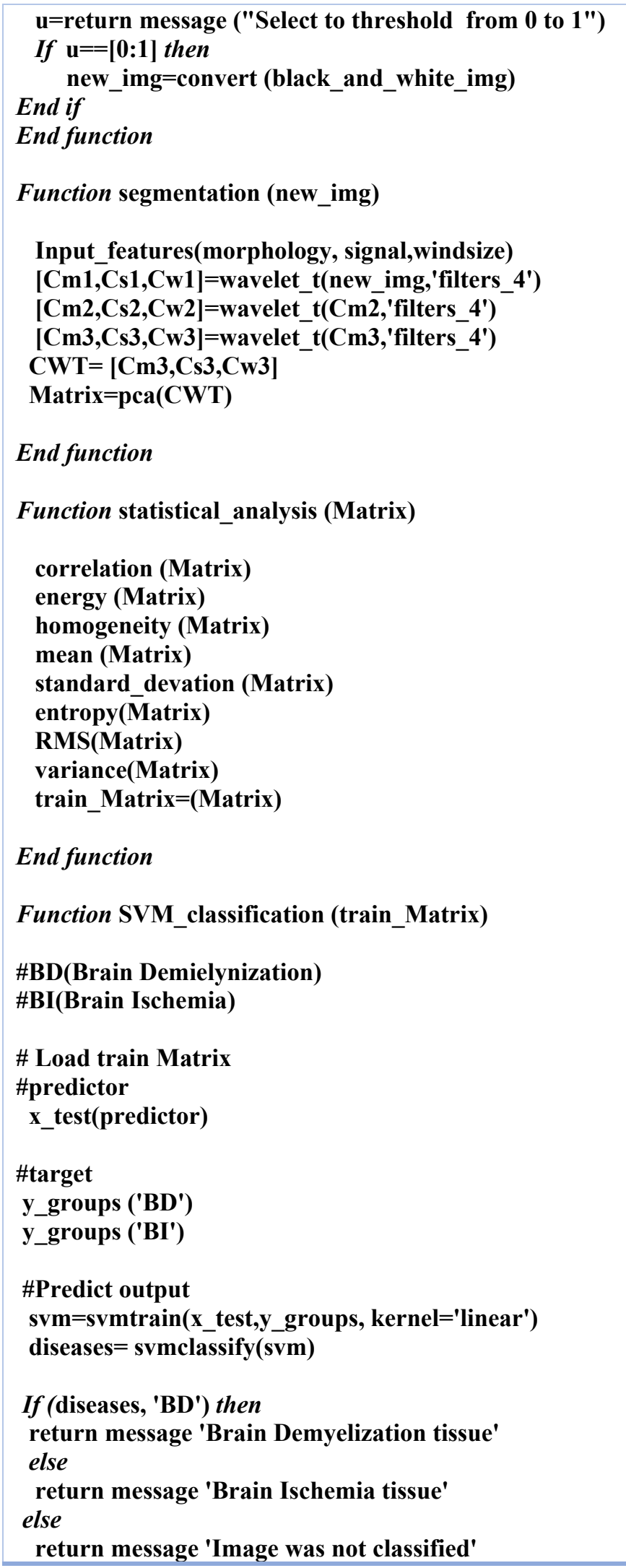




\section{End if \\ End function \\ End}

Segmentation supervised dataset and Supper vector machine learning

Segmentation is a process of partitioning a digital image into multiple segments or set of pixels. Segmentation is used to locate objects and boundaries in the image ${ }^{14}$. For the segmentation of the images there are many methods, in the work of Daniel García-Lorenzo ${ }^{14}$ this theme is widely descripted.

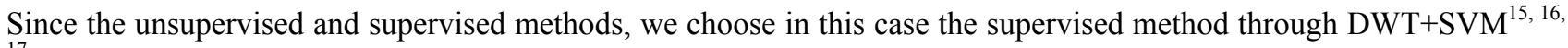
17 .

The kernel support vector machine (SVM) was used as diseases brain classification. It is a supervised machine learning algorithm which can be used for both classification and regression challenges ${ }^{15,18,19}$. In this work we used SVM for cerebral images classification problem, using the main characteristics of each diseases i.e. morphology, to identify which class (or group) BD or BI belongs. The characteristics of an object are typically presented in a vector $\overrightarrow{\boldsymbol{x}}$ whose output result is:

$$
y=f(\vec{w} \cdot \vec{x})=f\left(\sum_{j} w_{j} x_{j}\right)
$$

Where $\boldsymbol{w}$ is an actual vector of weights and $\boldsymbol{f}$ is a function that converts the point-to-point product of the two vectors into the desired output. The weight vector $\boldsymbol{w}$ learn from a set of training samples. Often $\boldsymbol{f}$ is a simple function that maps all values above a certain threshold to the first class and the remainder to the second class ${ }^{15,18}$.

For a two-class diseases, one can visualize the operation of a linear classifier as a partition of the high-dimensional input space with a hyperplane: all points on one side of the hyperplane will be classified as "BD", while the others will be classified as "BI".

As a result of submit brains images to the trained kernel SVM, the outputs prediction datasets were divided into groups the $\mathrm{BI}$ and $\mathrm{BD}$ tissue segmentation images classification strategy.

\section{RESULTS AND DISCUSSION}

\section{Database}

The database of all images utilized in this project firstly were anonymized and codified for the posterior processing and application in the algorithm, in that way, we care the patient confidentiality and privacy.

The series of images collected were classified in two groups; the normal images or different diseases and the group of images with the disease studied in this project.

First in our algorithm extract the six relevant images of each series and patient respectively. For propose of this projected we choose the T2-weighted fast spin-echo, and Fast-FLAIR according to the related works for detection of ischemia and demyelinating disease into brain magnetic resonance images ${ }^{1-7}$.

\section{Algorithm implementation: first stage}

The format of collected images from the series of patients were in *.dcm (dicom file), our algorithm in this step allow to read a dicom file or convert the dicom file in another format of image *.jpg, *png, *bmp, *tiff. The work with this formats of the images is more easily for implemented the different methodologies of segmentation and classification ${ }^{14,15}$. 
The process for identify the features of the diseases in this work is the next:

1. Convert the file image to gray scale.

2. In automatic case we can see and select the threshold for the convert the image in a binary format. There are different

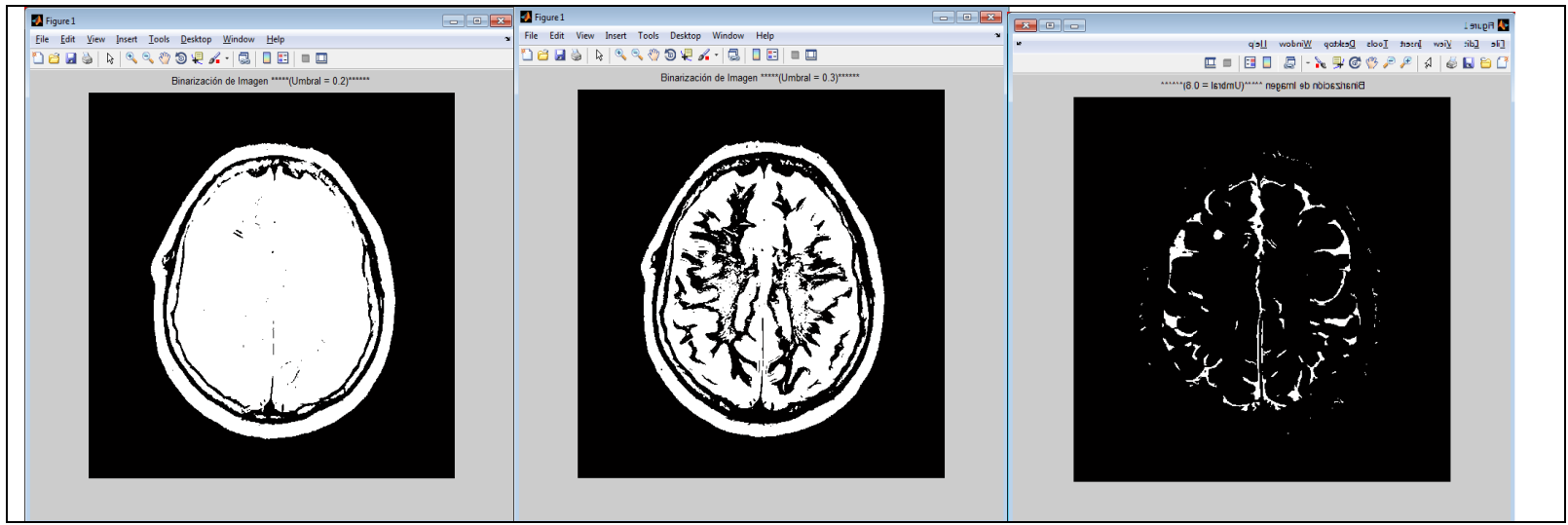

Figure 2. MRI images and the automatic process for select the threshold for to do the binary image.

3. Erosion the image for identify the features of demyelinating and ischemia. We try this with different functions in Matlab (i.e. imerode and streel functions) with the structuring element.

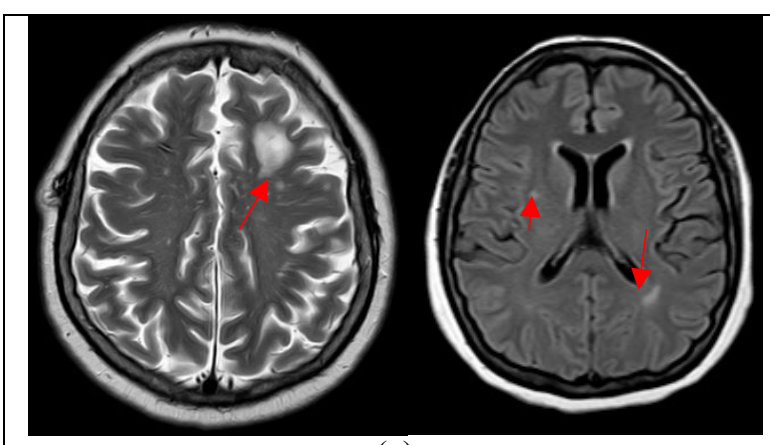

(a)

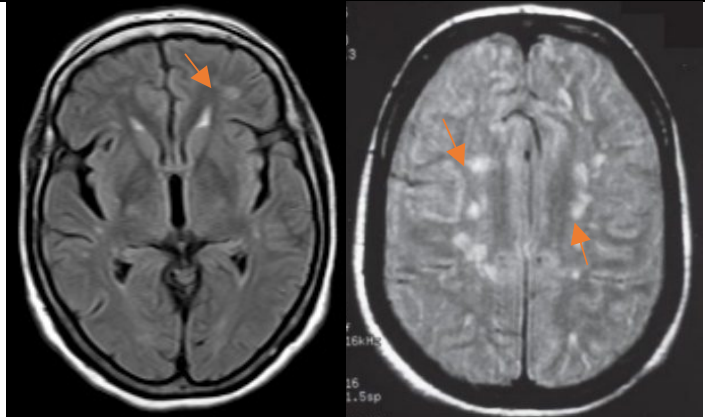

(b)

Figure 3. MRI images of (a) Ischemia brain disease (BI) and (b) Demyelinating disease (BD); before the processing. 


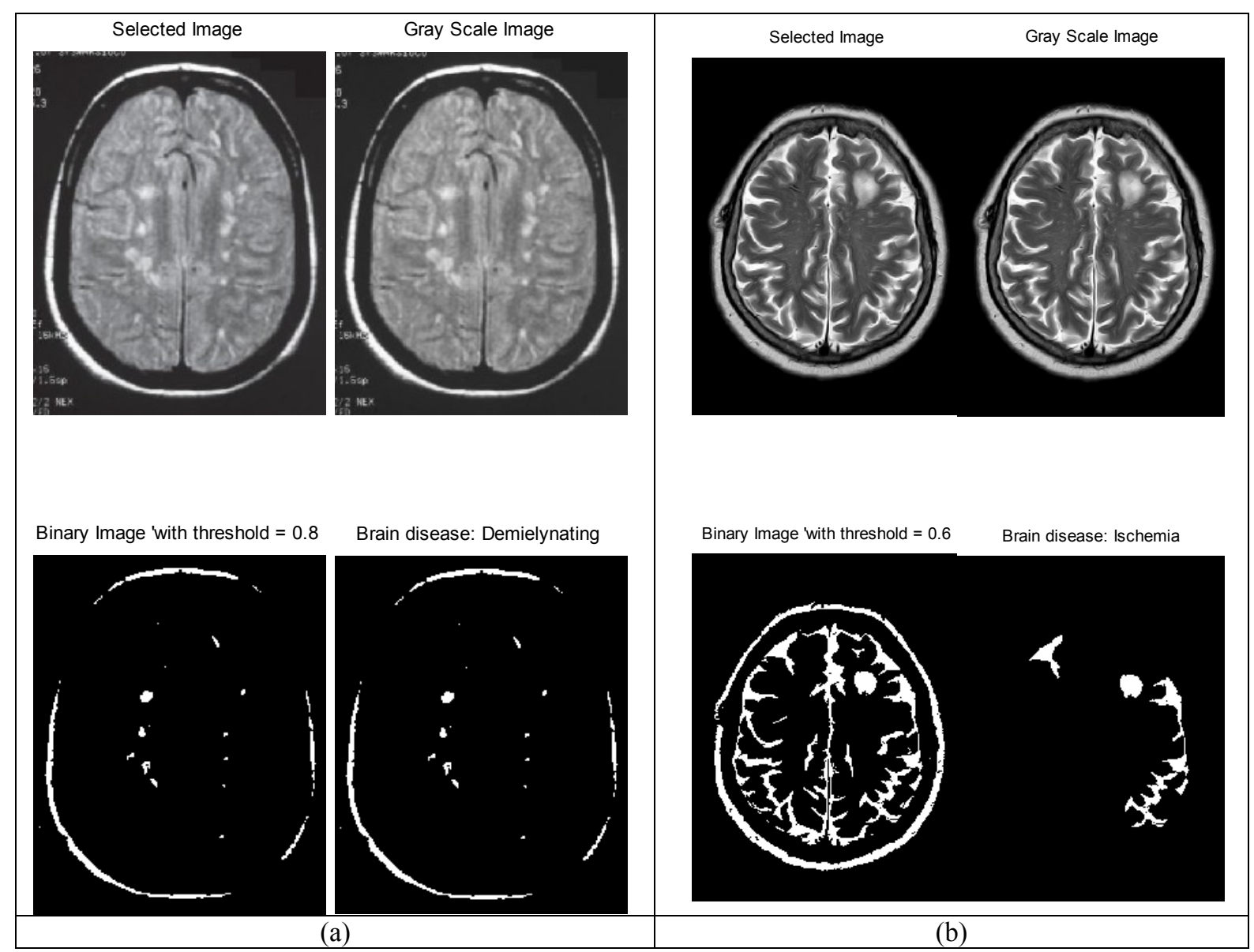

Figure 4. MRI images after the processing and detection of features for identify (a) Demyelinating brain disease (BI) and (b) Ischemia brain disease (BD).

We can see that for this stage of the algorithm, the features of the brain diseases object of our study until do not have a perfect definitions. Until this point the algorithm has the advantage to allow select the threshold for obtain a better classification, but also the weakness is that yet that features also require a good knowledge and ability to recognize the principal medical criteria for differentiate the diseases. For this work we use the medical criteria for recognize the disease accord to McDonald ${ }^{5}$.

For that reason and after the read of several works and methods, we decide that the best way to obtain a better extraction of features for these diseases is DWT+ SVM according with Zhang, Y and Wu, L. ${ }^{15}$, whose in their work applied this method for detect, classify and differentiate the benign and malignant.

However there are other methodologies and algorithms that uses a different automated approaches especially in order of the Multiple Sclerosis (MS) disease such as reported by LLado et al ${ }^{17-22}$. In the case of ischemic disease, there are not many works such the MS, but similar to the other works, these focus also in the extraction of the features of the infarct tissue ${ }^{1,3,4}$.

\section{CONCLUSIONS:}

In summary, we report the first stage of the algorithm: the design and the implementation of the first with a semiautomatic classification. The importance and accurate of the algorithm for identify one or another disease in the brain 
magnetic resonance images, is the form of the extract the features that define with the best fidelity according to the morphological medical criteria. For this first stage we only used different methods of erosion images with different structural operator for walk over the matrix images. In the next our paper we will report the all result of the algorithm using DWT + PCA and SVM.

\section{ACKNOWLEDGMENTS}

The authors are grateful with the Universidad Técnica Particular de Loja Hospital (H-UTPL) for collaborate with the data for this project.

\section{REFERENCES}

[1] Boltze, J., Kleinschnitz, C., Reymann, K. G., Reiser, G., Wagner, D. C., Kranz, A., \& Michalski, D. Neurovascular pathophysiology in cerebral ischemia, dementia and the ageing brain-current trends in basic, translational and clinical research. Experimental \& translational stroke medicine, 4(1), 14, 2012.

[2] Miller, D., Weinshenker, B., Filippi, M., Banwell, B., Cohen, J., Freedman, M., Polman, C. Differential diagnosis of suspected multiple sclerosis: a consensus approach. Multiple Sclerosis (Houndmills, Basingstoke, England), 14(9), 2008, 1157-1174. http://doi.org/10.1177/1352458508096878.

[3] Ona Wu, Toshihisa Sumii, Minoru Asahi, Masao Sasamata, Leif Østergaard, Bruce R Rosen, Eng H Lo, and Rick M Dijkhuizen. Infarct Prediction and Treatment Assessment with MRI-based Algorithms in Experimental Stroke Models J Cereb Blood Flow Metab January 2007 27: 196-204, doi:10.1038/sj.jcbfm.9600328.

[4] Pons Porrata LM, García Gómez O, Soto Infante V, González Ferro I. Valor de la resonancia magnética de imágenes en pacientes con enfermedad cerebrovascular isquémica [article on line]. MEDISAN 2009;13(2). $<$ http://bvs.sld.cu/revistas/san/vol13_2_09/san10209.htm>.

[5] Rovira, A., Tintoré, M., Álvarez-Cermeño, J. C., Izquierdo, G., \& Prieto, J. M. Recomendaciones para la utilización e interpretación de los estudios de resonancia magnética en la esclerosis múltiple. Neurología, 25(4), 248-265, 2010.

[6] Sanak, D., Horak, D., Herzig, R., Hlustik, P., \& Kanovsky, P. (2009). The role of magnetic resonance imaging for acute ischemic stroke. Biomedical Papers, 153(3), 181-187.

[7] Miller, D. H., Filippi, M., Fazekas, F., Frederiksen, J. L., Matthews, P. M., Montalban, X. and Polman, C. H. (2004), Role of magnetic resonance imaging within diagnostic criteria for multiple sclerosis. Ann Neurol., 56: 273278. doi:10.1002/ana.20156.

[8] Zhang, Y., L. Wu, and S. Wang, "Magnetic resonance brain image classification by an improved artificial bee colony algorithm," Progress In Electromagnetics Research, Vol. 116, 65 -79, 2011.

[9] Oikonomou, A., I. S. Karanasiou, and N. K. Uzunoglu, "Phased array nearfield radiometry for brain intracranial applications,"Progress In Electromagnetics Research, Vol. 109, 345- 360, 2010.

[10] Tillema J-M, Pirko I. Neuroradiological evaluation of demyelinating disease. Therapeutic Advances in Neurological Disorders. 2013;6(4):249-268. doi:10.1177/1756285613478870.

[11] Scapaticci, R., L. Di Donato, I. Catapano, and L. Crocco, A feasibility study on microwave imaging for brain stroke monitoring," Progress In Electromagnetics Research B, Vol. 40,305-324, 2012.

[12] Chaturvedi, C. M., V. P. Singh, P. Singh, P. Basu, M. Singaravel, R. K. Shukla, A. Dhawan, A. K. Pati, R. K. Gangwar, and S. P. Singh. $2.45 \mathrm{GHz}(\mathrm{CW})$ microwave irradiation alters circadian organization, spatial memory, DNA structure in the brain cells and blood cell counts of male mice, mus musculus," Progress In Electromagnetics Research B, Vol. 29, 23-42, 2011. 
[13] Chang T, Kelley RE. Ischemic Demyelination. In Neuroinflammation. Elsevier Inc.2011. p. 277-291. Available from, DOI: 10.1016/B978-0-12-384913-7.00013-7.

[14] Daniel García-Lorenzo, Simon Francis, Sridar Narayanan, Douglas L. Arnold, D. Louis Collins, Review of automatic segmentation methods of multiple sclerosis white matter lesions on conventional magnetic resonance imaging, Medical Image Analysis, Volume 17, Issue 1, January 2013, Pages 1-18, ISSN 1361-8415, http://dx.doi.org/10.1016/j.media.2012.09.004.

[15] Zhang, Y., \& Wu, L. An MR brain images classifier via principal component analysis and kernel support vector machine. Progress In Electromagnetics Research, 130, 369-388 (2012).

[16] R. C. Gonzales and R. E. Woods. Digital Image Processing. Prentice Hall, second edition, 2002. 795 pages, ISBN 0-201-18075-8.

[17] Emin Tagluk, M., M. Akin, and N. Sezgin, Classification of sleep apnea by using wavelet transform and artificial neural networks, Expert Systems with Applications, Vol. 37, No. 2, 1600-1607, 2010.

[18] Mariano Cabezas, Arnau Oliver, Xavier Lladó, Jordi Freixenet, Meritxell Bach Cuadra, A review of atlas-based segmentation for magnetic resonance brain images, Computer Methods and Programs in Biomedicine, Volume 104, Issue 3, December 2011, Pages e158-e177, ISSN 0169-2607, http://dx.doi.org/10.1016/j.cmpb.2011.07.015.

[19] Zhang, Y., L. Wu, and S. Wang, "Magnetic resonance brain image classification by an improved artificial bee colony algorithm," Progress In Electromagnetics Research, Vol. 116, 65 -79, 2011.

[20] Martijn D. Steenwijk, Petra J.W. Pouwels, Marita Daams, Jan Willem van Dalen, Matthan W.A. Caan, Edo Richard, Frederik Barkhof, Hugo Vrenken, Accurate white matter lesion segmentation by k nearest neighbor classification with tissue type priors (kNN-TTPs), NeuroImage: Clinical, Volume 3, 2013, Pages 462-469, ISSN 2213-1582, http://dx.doi.org/10.1016/j.nicl.2013.10.003.

[21] Xavier Lladó, Arnau Oliver, Mariano Cabezas, Jordi Freixenet, Joan C. Vilanova, Ana Quiles, Laia Valls, Lluís Ramió-Torrentà, Àlex Rovira, Segmentation of multiple sclerosis lesions in brain MRI: A review of automated approaches, Information Sciences, Volume 186, Issue 1, 1 March 2012, Pages 164-185, ISSN 0020-0255, http://dx.doi.org/10.1016/j.ins.2011.10.011.

[22] Hrishikesh Deshpande, Pierre Maurel, Christian Barillot, Classification of multiple sclerosis lesions using adaptive dictionary learning, Computerized Medical Imaging and Graphics, Volume 46, Part 1, December 2015, Pages 2-10, ISSN 0895-6111, http://dx.doi.org/10.1016/j.compmedimag.2015.05.003.

[23] Kevin C. Ma, James R. Fernandez, Lilyana Amezcua, Alex Lerner, Mark S. Shiroishi, Brent J. Liu, Design and development of an ethnically-diverse imaging informatics-based eFolder system for multiple sclerosis patients, Computerized Medical Imaging and Graphics, Volume 46, Part 2, December 2015, Pages 257-268, ISSN 08956111, http://dx.doi.org/10.1016/j.compmedimag.2015.09.007.

[24] Solomon, A. J., Schindler, M. K., Howard, D. B., Watts, R., Sati, P., Nickerson, J. P., \& Reich, D. S. “Central vessel sign" on 3T FLAIR* MRI for the differentiation of multiple sclerosis from migraine. Annals of Clinical and Translational Neurology, 3(2), 82-87. http://doi.org/10.1002/acn3.273, 2016.

[25] H. H. Barret. Foundations of Image Science. John Wiley \& Sons, New Jersey, U.K., third edition, 2004. 